\title{
Review of: "The tomato yellow leaf curl virus C4 protein alters the expression of plant developmental genes correlating to leaf upward cupping phenotype in tomato"
}

\section{Muhammad Tahir}

Potential competing interests: The author(s) declared that no potential competing interests exist.

The paper entitled "The tomato yellow leaf curl virus C4 protein alters the expression of plant developmental genes correlating to leaf upward cupping phenotype in tomato" has been reviewed. The manuscript is nicely written and approved for publication. 